
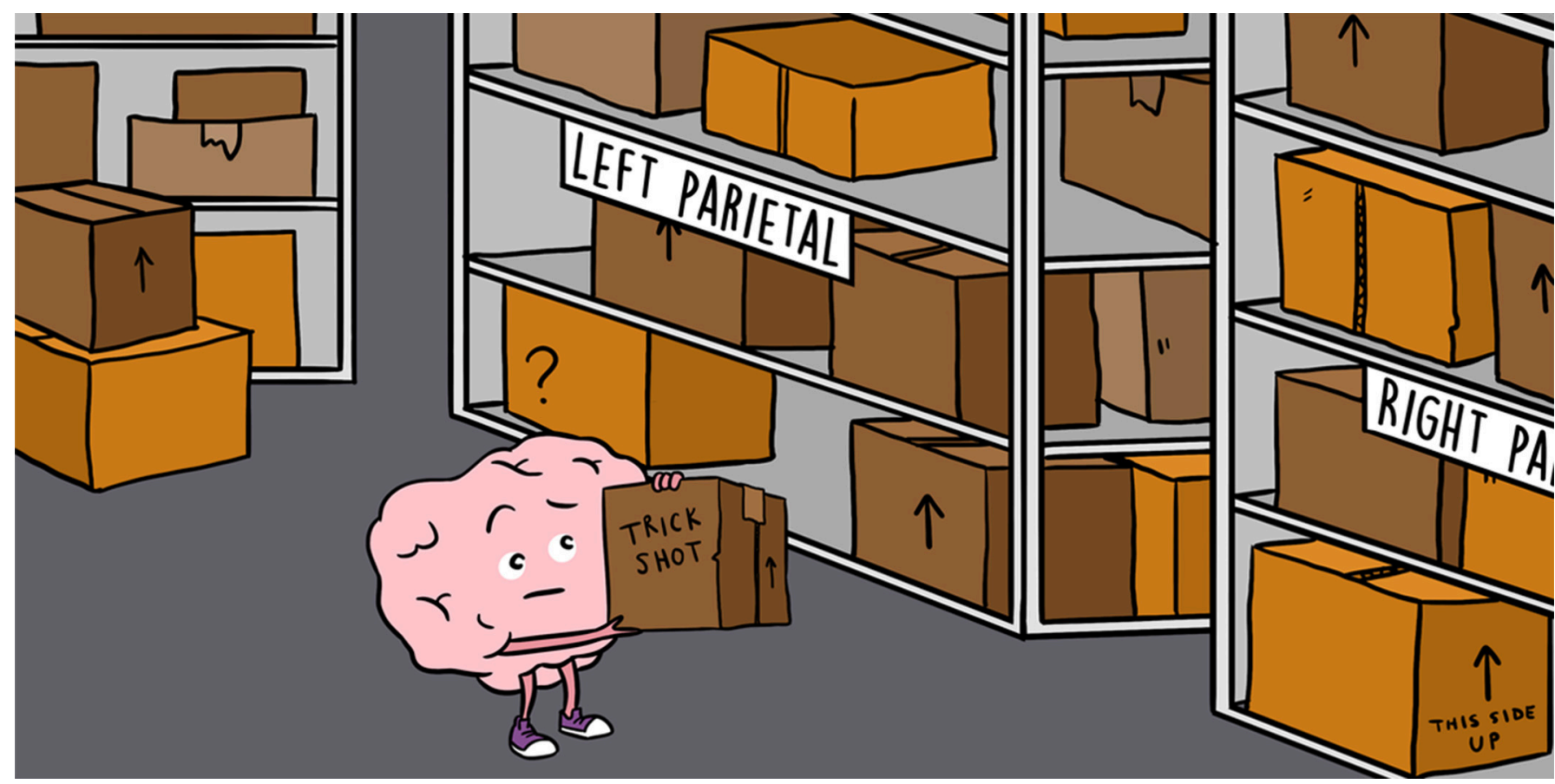

\title{
WHERE ARE MOTOR MEMORIES LOCATED IN THE BRAIN?
}

\section{Kathleen Y. Haaland ${ }^{1 *}$, Lee H. Stapp ${ }^{2}$ and Robert L. Sainburg ${ }^{3}$}

${ }^{1}$ Departments of Psychiatry \& Behavioral Sciences and Neurology, University of New Mexico, Albuquerque, NM, United States ${ }^{2}$ New Mexico VA Healthcare System, Albuquerque, NM, United States

${ }^{3}$ Departments of Kinesiology \& Neurology, The Penn State University, University Park, PA, United States

YOUNG REVIEWERS:

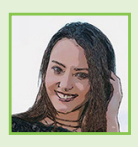

LÍVIA

AGE: 16

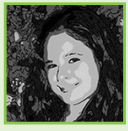

NICOLE

AGE: 15

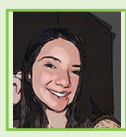

RAFAELA

AGE: 15

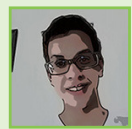

RAMON

AGE: 11

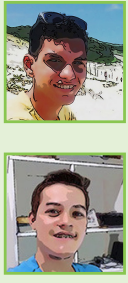

VICTOR AGE: 17 VINICIOS AGE: 16

When we practice a movement over and over again, we perform better, partly because we develop new motor memories, or representations, in our brains. We will not perform better if we cannot store motor representations in our brains. People who play sports or people who dance, for example, have to develop motor representations by repeating the same movements over and over, so they can perform them in different situations. The creation of better and better motor representations as we practice a movement is called motor adaptation. Motor representations are formed and stored in the brain, just like our memories of people and events. Motor representations for familiar movements, like brushing teeth, are located in a part of the brain called the left parietal lobe. We wanted to find out if motor representations for other movements are located in the left, but not the right, parietal lobe. So, we compared the motor adaptation of pointing responses in patients with left or right parietal lobe damage. We found that motor adaptation was impaired after 
MOTOR

REPRESENTATION

A memory of a movement that we use to make movements in the future. These representations or memories are created by groups of brain cells that interact to help you perform a movement you have learned. This memory is flexible and can be replayed to make movements in different situations, such as swinging a baseball bat in practice or in a game.

\section{MOTOR}

Movements that we make with our body or body parts.

\section{PARIETAL LOBE}

Each side (hemisphere) of our brain has four lobes: Frontal, Temporal, Parietal, and Occipital (see Figure 1), each with different functions. Our parietal lobes are important for processing sensations in our bodies, such as touch, movement, pain, and temperature and other functions not discussed in this paper. We also store our motor memories in our left parietal lobe.

\section{MOTOR}

\section{ADAPTATION}

The changes in our motor memory and motor performance that occur when we practice a movement over and over again.

\section{left, but not right, parietal lobe damage, suggesting that these motor representations are also located in the left parietal lobe.}

\section{MOTOR REPRESENTATIONS ARE IMPORTANT FOR SPORTS}

How do you learn a new sport? What would your parents or coach say? Practice, practice, practice! And as you practice, scientists think that you develop something called motor representations in your brain, which are like motor (movement) memories. Motor representations are created by groups of brain cells that interact to help you perform a movement you have learned. These representations allow you to perform better. They allow you to make the basket, slam the tennis ball, or play a violin concerto. Based upon what is happening on the soccer field, the star player can select the best response based upon her experience and the motor representations that have been developed and stored in her brain through practice. Check out this video for concrete examples of the increased speed and agility that comes with practice in cup stacking, a new Junior Olympics event https://www. youtube.com/watch?v=82DNYqurkxo.

\section{WHERE ARE MOTOR MEMORIES/REPRESENTATIONS LOCATED IN THE BRAIN?}

We know these motor representations are stored in the brain. But where in the brain?

One of the best ways to answer that question is to study patients with brain damage. Previous studies have shown that the motor representations for familiar movements, like brushing teeth, are located in an area of the brain called the left parietal lobe [1]. As seen in Figure 1, the parietal lobes are located on the sides of our brains. They process sensations from our body, such as touch, movement, pain, and temperature. They are also important for creating and storing motor memories, as well as some other things that we do not discuss in this paper. Motor adaptation is the term we use for learning a new movement skill. Motor adaptation was found to be impaired in two patients with parietal lobe damage. One patient had damage to the parietal lobes on both sides of the brain [2] and the other had damage to only the left parietal lobe [3]. However, because a patient with damage to only the right parietal lobe was not examined, we do not know if the left parietal lobe is more important than the right parietal lobe. Also, you never know if a single patient represents what happens in all people. So, to determine whether motor memories are created and stored in only one side of the brain or in both the left and the right parietal lobes, we studied motor adaptation in two groups of brain-damaged patients with right or left parietal lobe damage. We predicted that patients with left parietal lobe damage would have 
Figure 1

Side view of the left side of the brain, with labels showing each of the lobes of the brain. This study focused on measuring adaptation in patients with damage to the left or right parietal lobes.

Figure 2

(A) The machine that records arm movements while it presents the targets and visual feedback to people in the study. Participants sat facing a mirror that hides their arms. Movements were recorded using two sensors placed on the arm and hand of each participant. (B) A TV mounted above the mirror projected circles for a start position, a target, and a cursor. The cursor is a circle with an " $x$ " in the middle, which gives visual feedback about hand position as the hand moves to the target. The scientist could make the cursor move in a different direction than the hand moved (Adaptation Condition) or in the same direction as the hand moved (After Effect Condition).

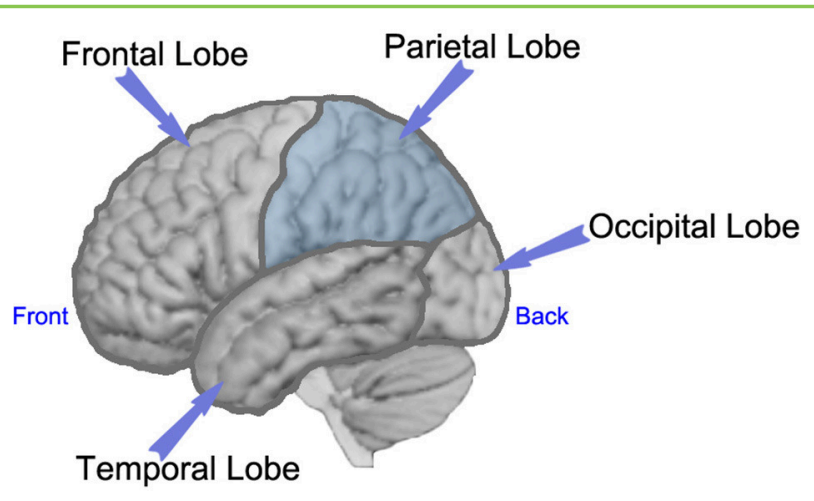

Figure 1

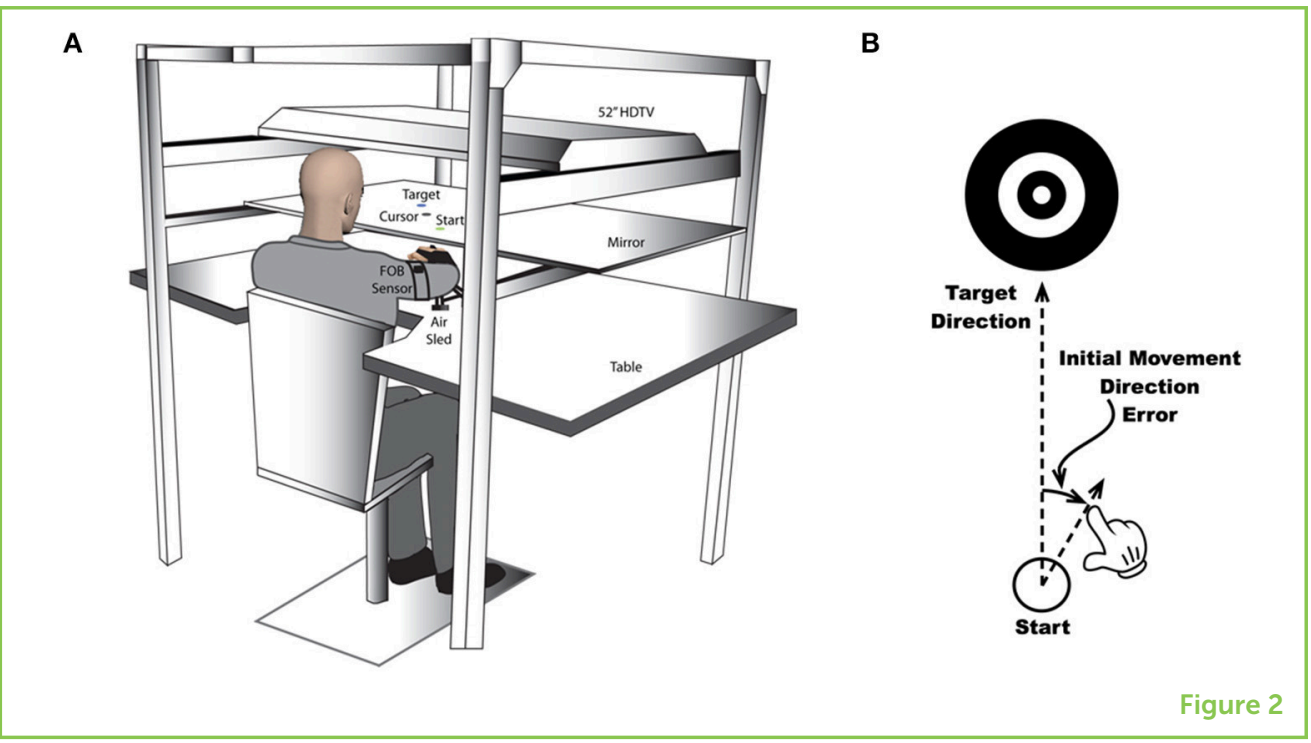

the most trouble with familiar movements, showing that many motor representations are stored only in the left parietal lobe.

\section{HOW DID WE TEST MOTOR ADAPTATION?}

To test motor adaptation, we asked everyone to sit in front of the machine shown in Figure 2. Imagine you are one of our participants. The goal is to learn to move the cursor from a start position to a target. You have to learn to do this because the cursor moves in a different direction than your hand. You have sensors on your arm that measure the direction your arm and hand move. Your arm is under the mirror so you cannot see it. All you see displayed on the mirror are circles for the start, the target, and the cursor. The cursor is a small circle with an " $x$ " in the middle, which gives you visual feedback about your hand position as you move from start to target. First, in the baseline condition, we give you correct visual feedback, meaning that we show your arm the way it actually moves. Second, in the adaptation condition, we give you visual feedback of your arm that is wrong. So, even when your arm is moving up and down, the visual feedback (cursor) shows your 


\section{MOVIE}

This movie shows what happens at the beginning, middle and end of adaptation to show learning. You can see the participant's hand and false visual feedback (cursor). At the beginning, the participant moves her hand vertically from start to target, but the visual feedback makes it look like she is moving to the left. By the end of adaptation, the participant has moved her hand to the right so the cursor moves straight toward the target. The adaptation shows that she has developed a new motor memory. Look at this movie a couple of times to get a better idea of how adaptation occurs. Available online at: https://youtu.be/ToyFcqx990.

\section{BRAIN HEMISPHERE}

Our brains have two sides or hemispheres (right and left) that look like mirror images of one another. For the most part, the left hemisphere controls the right side of the body and vice versa But, there are many things that are not exactly the same on each side of the brain. This is why some people are right handed and some are left handed and there are differences in what types of movement are controlled by each hand. arm moving to the left. You would adapt by gradually moving your arm to the right in order to make the visual feedback (cursor) go vertical. With practice you learn to adjust the direction of your movement to accurately hit the target, despite the bad feedback.

Look at this movie (https://youtu.be/To-yFcqx990) a couple of times to get a better idea of how adaptation occurs during the beginning, middle and end of the adaptation condition.

After adaptation, we begin the after effects condition, in which visual feedback is again accurate. If your brain is healthy, you would have developed a new motor representation based on the incorrect feedback during the adaptation condition, and you would continue to move your arm to the right a few times in the aftereffects session. A common motor after effect is "sea legs," which occurs when you spend time on a boat. When you get off the boat you wobble and sway, as though you were still on the moving boat. This happens because your brain is still compensating for the boat's sway. It is called an "after effect" because it occurs when it is no longer useful. In the motor task in our experiment, the after effect was that the participants aimed their hands in the wrong direction (to the right), as though the incorrect visual feedback were still present, even though it was not.

\section{WHO DID WE TEST?}

We tested two groups of brain-damaged stroke patients (five in each group) and two normal control groups, without brain damage (seven in each group). All participants were right handed and of a similar age. Because each side of the brain has a stronger influence on the opposite arm, we examined brain hemisphere or the arm opposite the hemisphere damaged for both stroke groups [right arm for the left parietal damaged group (LPD), left arm for right parietal damaged group (RPD)]. Also, because all participants were right handed, we compared each stroke group with a control group using the same arm [right arm for LPD and right normal control group (RNC); left arm for RPD and left normal control group (LNC)], to ensure that right hand preference did not influence our findings.

\section{WHAT DID WE FIND?}

Figure $3 \mathrm{~A}$ shows that all four groups were performing the same at the beginning of the adaptation condition. The two control groups, RNC and LNC, and the RPD group showed similar adaptation over the session. The RPD group's learning was normal. However, the LPD group did not show adaptation-they did not change the movement of their arms as much as the other three groups.

We saw the same pattern in the aftereffects data (Figure 3B). Again, it was only the LPD group, not the RPD group that did not show an 
Figure 3

(A) Shows the errors in the direction of movements when people are first exposed to the motor task. Each cycle (x axis) represents eight consecutive movements. In cycle 1 , when participants were first exposed to the task, participants make a $30^{\circ}$ error, and as they practice, the error goes down. Both control groups (blue) and the Right Parietal stroke group (A, left) adapt to the task the same. In contrast, the left Parietal stroke group (red, A right) does not decrease their error through training. (B) Shows the errors that occur when the rotation of the visual feedback is turned off, referred to as after-effects. The three groups that adapted to the rotation (LNC, RNC, and RPD) show large after effects, while LPD patients do not.

\section{HEMISPHERE}

DAMAGE (RIGHT OR LEFT)

Stroke blocks the blood flow to certain brain regions, which results in damage to those regions due to the lack of oxygen and nutrients. In this paper, we examine patients with damage to the parietal lobe. Therefore, we refer to this damage as RPD, or right parietal damage when it is in the right side of the brain, and LPD, or left parietal damage when it is in the left side of the brain.

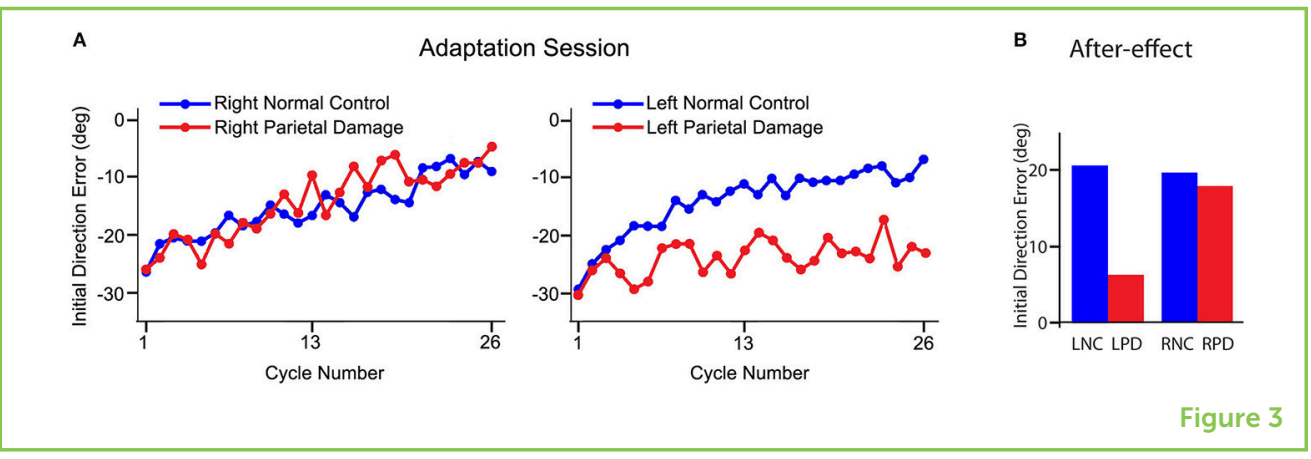

after effect, which supports the conclusion that the LPD group did not develop a new motor representation during adaptation. And again, the RPD group and the two normal control groups performed normally, showing that they developed new motor representations.

\section{WHAT DOES THIS STUDY REALLY MEAN?}

This is the first group study of brain-damaged patients showing that the left parietal lobe is more important than the right parietal lobe for learning to adapt to inaccurate visual feedback. Scientists think that this poor learning is due to a decreased ability to develop new motor representations or memories, which is supported by our after-effect data. These findings are important first because this is a group study of motor adaptation in brain-damaged patients. Science cannot rely on single cases because single cases might not represent what happens in everyone. So, group studies are necessary to confirm what is seen in the study of individual cases. Second, we found that the left parietal lobe is critical for motor adaptation. We examined groups of patients with left or right parietal damage, while the case reports examined single patients with left parietal damage or parietal damage on both sides $[2,3]$. Neither of those single-case reports examined a patient with right parietal damage only. Our work suggests that the left parietal lobe, but not the right, is the site of motor representations for motor adaptation. Other experiments have found that people with left, but not right parietal lobe damage have difficulties with familiar movements, like brushing their teeth, and cutting their food with a knife. This makes sense, because all movements must adapt to different task conditions. For example, you can brush your teeth whether you are standing or lying on your back, even though these conditions require different forces and movements.

Of course, more research is necessary to find out which other areas of the brain are involved in motor adaptation and motor learning. Most complex skills are dependent on many different brain areas that may have different functions in a task. However, these areas must all talk to each other in order to produce accurate motor performance. 


\section{HOW CAN THESE RESULTS BE USED IN THE REAL WORLD?}

If our results are true not just for our experimental task, but for everyday movements, like learning how to type, or brush your teeth, or flip a pancake, then a technique called brain stimulation might be used to help create motor representations in patients with brain damage who cannot do these everyday tasks. Brain stimulation techniques involve the placement of electrical stimulators on the scalp that can stimulate the brain without surgery. Scientists are now exploring whether such stimulation can help brain-damaged patients to overcome motor deficits and help people without brain damage to learn better. However, scientists need to know the best part of the brain to stimulate. Our results suggest that stimulation of the left parietal lobe might enhance motor learning in both brain-damaged patients and people without brain damage.

Most recently, scientists have found a way to use the activity from the left parietal lobe of a patient to control his prosthetic arm. This patient had a spinal cord injury that prevented his brain from controlling his arms and legs [4]. The scientists used the activity of cells in the parietal lobe that help with complex movements to allow this patient to pick up a beer and drink it by himself ... for the first time since his spinal cord injury. So, sometimes even when science seems like it is interesting, but not useful, real-world applications develop over time. There are many ways the present findings could be used in the real world. Can you think of more?

\section{ORIGINAL SOURCE ARTICLE}

Mutha, P. K., Sainburg, R. L., and Haaland, K. Y. 2011. Left parietal regions are critical for adaptive visuomotor control. J. Neurosci. 31:6972-81. doi: 10.1523/JNEUROSCI.6432-10.2011

\section{REFERENCES}

1. Vingerhoets, G. 2014. Contribution of the posterior parietal cortex in reaching, grasping, and using objects and tools. Front. Psychol. 5:151. doi: 10.3389/fpsyg.2014.00151

2. Newport, R., Brown, L., Husain, M., Mort, D., and Jackson, S. R. 2006. The role of the posterior parietal lobe in prism adaptation: Failure to adapt to optical prisms in a patient with bilateral damage to posterior parietal cortex. Cortex. 42:720-9. doi: 10.1016/S0010-9452(08)70410-6

3. Wolpert, D. M., Goodbody, S. J., and Husain, M. 1998. Maintaining internal representations: the role of the human superior parietal lobe. Nat. Neurosci. 1:529-33.

4. Aflalo, T., Kellis, S., Klaes, C., Lee, B., Shi, Y., Pejsa, K., et al. 2015. Decoding motor imagery from the posterior parietal cortex of a tetraplegic human. Science. 348:906-10. doi: 10.1126/science.aaa5417 
SUBMITTED: 18 July 2018; ACCEPTED: 20 March 2019;

PUBLISHED ONLINE: 08 May 2019.

EDITED BY: Sabine Kastner, Princeton University, United States

CITATION: Haaland KY, Stapp LH and Sainburg RL (2019) Where Are Motor Memories Located in the Brain? Front. Young Minds 7:54. doi: 10.3389/frym.2019. 00054

CONFLICT OF INTEREST STATEMENT: The authors declare that the research was conducted in the absence of any commercial or financial relationships that could be construed as a potential conflict of interest.

COPYRIGHT @ 2019 Haaland, Stapp and Sainburg. This is an open-access article distributed under the terms of the Creative Commons Attribution License (CC BY). The use, distribution or reproduction in other forums is permitted, provided the original author(s) and the copyright owner(s) are credited and that the original publication in this journal is cited, in accordance with accepted academic practice. No use, distribution or reproduction is permitted which does not comply with these terms.

\section{YOUNG REVIEWERS}

LÍVIA, AGE: 16

I am 16 years old. I like sports very much but dance is my favorite hobby. I also play violin since 8 years old. My dream is to become a medical doctor and, in the future, work for doctors without borders.

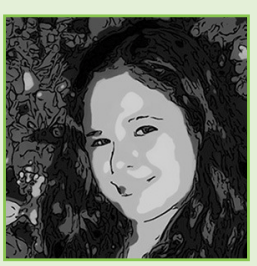

\section{NICOLE, AGE: 15}

I study at Santo André School and my favorite subjects are English, Chemistry, Portuguese, and Biology. In my free time I usually listen to music, read and watch series and movies on TV. I also love cooking. I intend to study pharmacy at University.

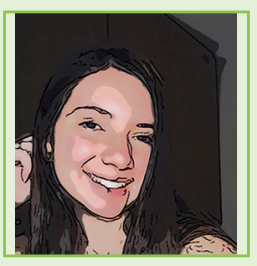

\section{RAFAELA, AGE: 15}

I study at Santo André School at Jaboticabal. In my free time I enjoy reading and I love traveling.

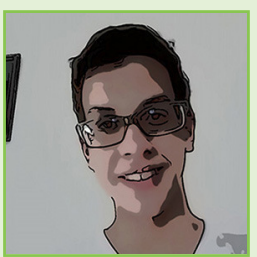

\section{RAMON, AGE: 11}

I study in the first grade of Santo André High School at Jaboticabal city. I really like to play online games, but I also like to study, listen to music and hang out with friends. My life is always hectic, and I love it. 

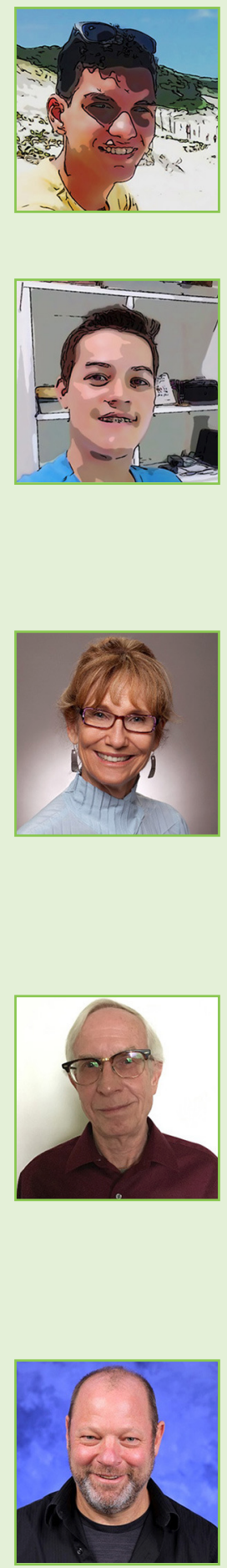

VICTOR, AGE: 17

I study at Santo André School. I like to study English, and have the opportunity to interact with the world. I intend to study law at University.

\section{VINICIOS, AGE: 16}

I study at Santo André High School and I would like to become a Civil Engineer.

\section{AUTHORS}

\section{KATHLEEN Y. HAALAND}

I am a clinical neuropsychologist and professor at the University of New Mexico. I evaluate patients with thinking problems to diagnose problems like Alzheimer's disease. My research focuses on which parts of the brain are important for movement and on how the two sides of the brain control different movements. My research compares movement problems in patients with damage to different parts of the left and right sides of the brain, like in this article. I like to hike, read, cook, and talk, especially about politics. *khaaland@unm.edu

\section{LEE H. STAPP}

I work in a Neuropsychology Clinic in the VA Health Care System. I give various tests to patients to help see what specific thinking problems they have. For many years I was a research assistant helping study how the brain controls movement. I helped examine patients' behavior and their brain images for comparison with healthy people without brain damage. This allows us to study people with damage in different areas of the brain, as in this article. I like to read, listen to music, and visit with my daughter frequently.

\section{ROBERT L. SAINBURG}

My name is Bob Sainburg. I am a Professor of Kinesiology and Neurology at Penn State University, and I am an occupational therapist. I study how the brain controls movement, and have a special interest in how each side of the brain differs in control of movement, and how that results in handedness. I also study how movement control is affected by stroke and I am developing new rehabilitation techniques to help stroke patients recover movement control. I like running, hiking, and biking in my free time. 